\title{
Influence of Surface Roughness on Interdiffusion Processes in InGaP/Ge Heteroepitaxial Thin Films
}

\author{
P. Joice Sophia, a,z G. Attolini, ${ }^{a}$ M. Bosi, ${ }^{a}$ E. Buffagni, ${ }^{a}$ C. Ferrari, ${ }^{a}$ C. Frigeri, ${ }^{a}$ K. Vad, ${ }^{b}$ \\ A. Csik, ${ }^{b}$ V. Takáts, ${ }^{\text {b }}$ and Z. Zolnaic \\ a Institute of Materials for Electronics and Magnetism, National Research Council, Parma, Italy \\ ${ }^{b}$ Institute for Nuclear Research, Hungarian Academy of Sciences, Debrecen, Hungary \\ ${ }^{\mathrm{c}}$ Research Centre for Natural Sciences, Institute of Technical Physics and Materials Science, \\ Hungarian Academy of Sciences, Budapest, Hungary
}

\begin{abstract}
In the present work, we report on the study of $\mathrm{InGaP} / \mathrm{Ge}$ heterojunctions grown by metal organic vapor phase epitaxy at different growth temperatures, with the aim of analyzing properties of the layer and interface between InGaP epilayer and germanium substrate. Secondary Neutral Mass Spectroscopy, Rutherford Backscattering Spectrometry, High Resolution X-Ray Diffraction, Transmission Electron Microscopy and Atomic Force Microscopy have been used to characterize the layers. The main goal of this work is to get information about diffusion processes of $\mathrm{Ga}, \mathrm{In}, \mathrm{P}$ in the substrate and of $\mathrm{Ge}$ in the epitaxial film. Since the interface roughness during sputtering and the effect of diffusion depends on the growth temperature, depth profiles measured experimentally were combined with surface roughness data in order to get more reliable information about diffusion profiles and the real depth distribution of elements in the interface.
\end{abstract}

(C) 2014 The Electrochemical Society. [DOI: 10.1149/2.0021503jss] All rights reserved.

Manuscript submitted October 14, 2014; revised manuscript received November 2, 2014. Published 00 0, 2014.

Indium gallium phosphide ( $\mathrm{InGaP}$ ) is the fundamental material for many electronic and optical devices ${ }^{1}$ such as heterojunction bipolar transistors, metal oxide semiconductor field effect transistors (MOSFETs), light emitting diodes (LEDs), lasers and multi-junction solar cells. ${ }^{2-6}$ The properties of InGaP and germanium $(\mathrm{Ge})$ make them interesting materials for diffusion studies. Hence, the knowledge of diffusion mechanisms is significant in order to understand the different processes involved in the interface. Germanium junctions in high efficiency solar cells are usually realized by diffusion between the substrates and epitaxial GaAs or InGaP layers. ${ }^{7}$ For example, deposition of a GaAs layer on n-type germanium substrate ${ }^{8}$ can result in p-n junction due to higher concentration of gallium $(\mathrm{Ga})$ in the topmost part of the Ge substrate. On the other hand, $\mathrm{InGaP}$ deposition on p-type germanium substrate produces $n-p$ junction due to the different diffusion process between phosphorous (P), indium (In) and $\mathrm{Ga}$ elements, resulting in a top layer doped highly with $P$.

In spite of its large application capability, this compound suffers problems connected with the lattice mismatch between the epitaxial/binary/ternary compounds and germanium substrate. Spontaneous formation of ordered phase and different surface morphology can be due to the different growth conditions. ${ }^{9}$ The poor surface quality is a critical factor for junction abruptness. Moreover, the ordering process occurs through the formation of a mosaic of ordered domains dispersed in disordered matrix that cause materials inhomogeneity, which negatively affects the device performance.

In the present paper, we performed the study of InGaP/Ge thin films grown by low pressure metal organic vapor phase epitaxy (MOVPE) and were characterized from the interdiffusion point of view, in particular, the role of the surface morphology and roughness in the real depth profile determination. This work gives a detailed insight into the diffusion profiles of the film/substrate interface induced by the growth temperature.

\section{Experimental}

The InGaP films were grown on Ge (001) substrates by MOVPE technique in a horizontal reactor without substrate rotation at different growth temperatures of 600,650 and $675^{\circ} \mathrm{C}$ with a pressure of $60 \mathrm{mbar}$. The precursors used were trimethylgallium (TMG) and trimethylindium (TMI) for the elements of group III and phosphine $\left(\mathrm{PH}_{3}\right)$ in a $10 \%$ mixture of hydrogen for the group $\mathrm{V}$ element. In a typical growth experiment, the Ge substrate was thermally etched at $650^{\circ} \mathrm{C}$ for 5 minutes in order to remove the native oxide prior to introduction of the precursors into the reactor chamber. In order to obtain the same composition for all the samples, the ratio of $\mathrm{In} / \mathrm{Ga}$ was varied from 1.1 to 1.4 , while the ratio of V/III groups elements ranged from 95 to 87 . The InGaP was not intentionally doped.

Secondary Neutral Mass Spectroscopy (SNMS, type INA-X, SPECS GmbH) in the direct bombardment mode using $\mathrm{Ar}^{+}$ions with a fairly low energy for sputtering $\left(\mathrm{E}_{\mathrm{Ar}+}=350 \mathrm{eV}\right)$ was employed. The erosion area was confined to a circle of $2 \mathrm{~mm}$ in diameter by means of a tantalum (Ta) mask. The lateral homogeneity of the ion bombardment was checked by a profilometric analysis of sputtered craters. Rutherford Backscattering Spectrometry in combination with Channeling (RBS/C) analysis was performed in a scattering chamber with a two-axis goniometer connected to a $5 \mathrm{MV}$ Van de Graaff accelerator. The $1 \mathrm{MeV}^{4} \mathrm{He}^{+}$analyzing ion beam was collimated with two sets of four-sector slits with the dimensions of $0.5 \times 0.5 \mathrm{~mm}^{2}$, while the beam divergence was kept below $0.06^{\circ}$. The beam current was measured by a transmission Faraday cup. ${ }^{10}$ In the scattering chamber, the vacuum measurement was about $10^{-4} \mathrm{~Pa}$. In order to reduce the hydrocarbon deposition, liquid $\mathrm{N}_{2}$ cooled traps were used along the beam path and around the wall of the chamber. Backscattered $\mathrm{He}^{+}$ ions were detected using an ORTEC surface barrier detector mounted in Cornell geometry at scattering angle of $\theta=165^{\circ}$. The energy resolution of the detection system was $16 \mathrm{keV}$. Atomic Force Microscopy (AFM) in contact mode (Digital Instruments Nanoscope IIIa) was employed to study the surface morphology. The average surface roughness values were estimated from the analysis of AFM images. AFM measurements were performed over $15 \times 15 \mu \mathrm{m}^{2}$ areas on four different randomly selected places of the sample surface and the AFM image analysis was carried out by Gwyddion software. Transmission Electron Microscopy (TEM) operated in two-beam diffraction contrast and high resolution (HR-TEM) modes, using a JEOL 2200FS TEM/STEM field emission gun at $200 \mathrm{kV}$ was employed.

\section{Results and Discussion}

In order to estimate the composition of the layer, the mismatch $(\square \mathrm{d} / \mathrm{d}$ ) was determined from X-ray diffraction profiles recorded for the three samples grown at different temperatures $\left(600,650\right.$ and $\left.675^{\circ} \mathrm{C}\right)$. We used the first order formula $\square \mathrm{d} / \mathrm{d}=-\square \theta / \tan \theta$ to obtain the average indium content of $0.43\left(\operatorname{In}_{0.43} \mathrm{Ga}_{0.57} \mathrm{P}\right)$. In addition, the X-ray measurements showed that the layers were crystalline with defects inside the lattice and reciprocal lattice map of the same sample in 115 geometry indicated the presence of strain release.

AFM images of the samples grown at three different temperatures were presented in Fig. 1. Average surface roughness values were

\section{2} 63 64 104 

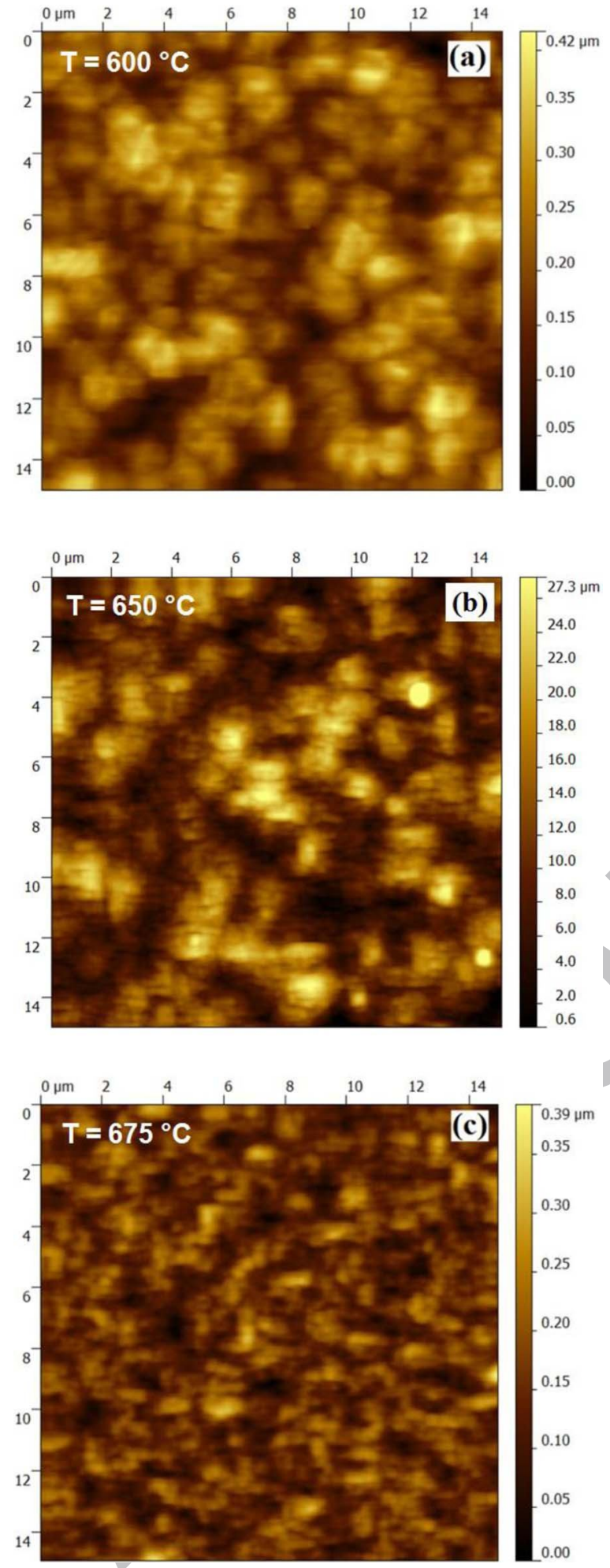

Figure 1. AFM images of InGaP layers grown on germanium substrates at a) $600^{\circ} \mathrm{C}$, b) $650^{\circ} \mathrm{C}$ and c) $675^{\circ} \mathrm{C}$.

105 determined from large image areas $\left(15 \times 15 \mu \mathrm{m}^{2}\right)$ and it was assured 106 that the scan area was always large enough to yield a representative 107 roughness value for the whole sample. AFM measurements show that 108 the average surface roughness of the samples decreases from about 10942 to $20 \mathrm{~nm}$ with the increase in the growth temperature from 600 to ${ }_{110} 675^{\circ} \mathrm{C}$ (Table I).

\begin{tabular}{lccc}
\multicolumn{3}{c}{ Table I. Roughness and Interface width vs Growth Temperatures. } \\
Sample & $\begin{array}{c}\text { Growth } \\
\text { temperature }\left({ }^{\circ} \mathrm{C}\right)\end{array}$ & $\begin{array}{c}\text { Average surface } \\
\text { roughness }(\mathrm{nm})^{*}\end{array}$ & $\begin{array}{c}\text { Interface width } \\
(\mathrm{nm})^{* *}\end{array}$ \\
\hline 1 & 600 & $\sim 42$ & 195 \\
2 & 650 & $\sim 32$ & 60 \\
3 & 675 & $\sim 20$ & 45 \\
*Determined from AFM. & & \\
${ }^{* *}$ Determined from SNMS. & &
\end{tabular}

The diffusion of constituents of the InGaP layer into Ge substrate 111 was checked by RBS measurements. Fig. 2 shows the RBS/C spectra 112 for two samples where the $\mathrm{InGaP}$ layer was chemically removed using 113 $\mathrm{HCl}$ solution from the substrate prior to measurements in order to have 114 only the contribution of the elements In, Ga, P diffused into the Ge substrates during growth time. Among elements, the background free ${ }_{116}$ detection was only possible for In, while the Ga signal was detected 117 very close to Ge (see Fig. 2). The detection limit was the highest for ${ }_{118}$ $\mathrm{P}$ due to its low atomic number and backscattering cross section. The 11 presence of about 1-3 at\% In can be observed in the subsurface region 120 of the samples. The higher amount of In diffused into the substrate of Sample 1 can be explained by an intensive grain boundary diffusion in the twin crystal structure which characterizes the $600^{\circ} \mathrm{C}$ growth ${ }_{123}$ mechanism, see the AFM patterns in Fig. 1a and 1c. It is difficult to 124 estimate the presence of $\mathrm{Ga}$ and $\mathrm{P}$ due to overlapping with $\mathrm{Ge}$ and ${ }_{125}$ the high detection limit compared to In. The channelling spectrum for the sample grown at $650^{\circ} \mathrm{C}$ showed a minimum yield of about $5 \%$ indicating a good crystalline quality for the Ge substrate.

Depth distribution of constituents determines the physical properties of thin films and has an important role in doping of Ge substrate, particularly in study of the infrared cell doping behavior. According to chemical reactions of CVD technology, the substrates are usually kept at high temperatures during sample preparation. As this time is long enough to let any atomic motion or diffusion between the substrate and film to take place, it is very important to determine the in-depth chemical composition, mainly at the interface. Since the main elements composition of a film determine the basic properties, the diffusion from the overlayer through the substrate during preparation time can modify these basic properties, as it is practically a doping process. In our previous works,, 911 we have already shown that a sputter based depth profile analysis is a unique tool for revealing interface

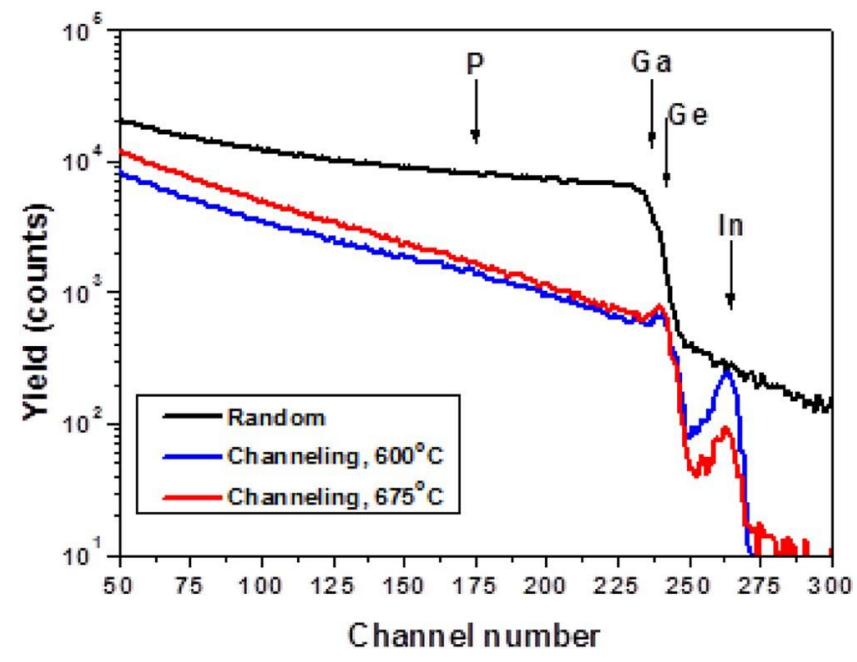

Figure 2. RBS channeling spectra of two substrates after removal of the InGaP layers grown at different temperatures of 600 and $675^{\circ} \mathrm{C}$. For comparison a random spectrum is also shown. Surface peak positions for $\mathrm{In}, \mathrm{Ga}, \mathrm{Ge}$, and P are represented by the arrows. 
142

143

144

structures. However, the depth resolution determining the quality of such measurements depends strongly on the roughness of the sputtered surface which is the bottom of the crater. Moreover, this roughness can change during sputtering. Since the depth profile analysis of a sample starts at the surface, the high surface roughness plays a crucial role in determination of depth resolution. In case of high surface roughness (e.g. $250 \mathrm{~nm}$ peak-to-valley value of Sample 1), it is unexpected that the surface becomes smooth at the end of sputtering. Even f the roughness decreases by sputtering, the thickness of the layer is not high enough to obtain finally a smooth surface. In this way, the interface roughness is expected to be high when the surface roughness is high. Here the interface roughness is the real surface roughness at the bottom of the crater during sputtering through the interface region between the film and substrate. At high interface roughness, the interface width is mainly determined by the roughness independent of sputtering processes and it is high as well. In contrary to this, the interface width is small when the interface surface is smooth, i.e. the sample surface is smooth. In general, the definition of interface width is arbitrary and phenomenological. Experimentally, it is usually given by the difference between the depth coordinates belong to $10 \%$ and $90 \%$ intensities of the analysed element. This is similar to the definition of depth resolution at a sharp interface. ${ }^{12}$ In Fig. 3a we have shown the method for determining the interface width.

In our experiments, from the surface analyses it turned out that our samples had high surface roughness depending on the growth temperature (Table I). As the growth temperature increases, the roughness decreases. The depth distribution of elements was revealed by SNMS echnique. Fig. 3 shows the trend of elements in InGaP layers grown at 600,650 and $675^{\circ} \mathrm{C}$ with the layer thickness of about $400 \mathrm{~nm}$. As it can be seen, the interface width decreases with the decrease of the surface roughness. According to Table I, the surface roughness of the sample grown at $675^{\circ} \mathrm{C}$ much higher than that is of the sample grown at $600^{\circ} \mathrm{C}$.

From the point of surface roughness, the depth profile analysis of $A / B$ interface can be critical. Due to sputtering mechanism, the elements of A and B can be mixed by the sputtering itself even they were not mixed before. In this manner, the atomic mixing means that both the elements are sputtered at the same time. This type of atomic mixing can be evoked by ion bombardment, crater shape and surface (interface) roughness. The main goal of this work is to get information about diffusion processes of $\mathrm{Ga}$, In, $\mathrm{P}$ in the substrate and of germanium in the film. However, it is not simple to make a distinction between the processes of diffusion mechanism and surface roughness with only depth profile analysis. A sharp change in concentration at a rough surface/interface can result in the same effect as a diffuse change in concentration at a smooth surface/interface. ${ }^{13}$ Both phenomena result in the same depth distribution of elements which can be characterized by the error function. In order to get information about the surface roughness contribution to the depth distribution of elements, we supplemented our SNMS measurements with calculaions of element depth distribution induced by the surface roughness. If the depth distribution measured experimentally differs from this calculated one, the deviation is caused by diffusion. Moreover, in a sputter depth profile analysis this deviation solely gives the real information about diffusion process.

The surface roughness determined experimentally was approached by a Gaussian distribution similar to that reported previously. ${ }^{12}$ Using this measured value and assuming a Gauss type depth distribution of the surface roughness, the depth concentration of elements can be calculated. If the calculated value fit with the experimental one, the depth distribution was only determined by the surface roughness, if not, it was governed by other physical process, e.g. by diffusion. Consequently, the difference between the calculated and experimentally measured concentration profiles refers to diffusion. Fig. 4 shows the In and Ge depth distributions at two growth temperatures $\left(650^{\circ} \mathrm{C}\right.$ and $675^{\circ} \mathrm{C}$ ) measured by SNMS. As it can be seen, the measured values of Ge intensity in the interface region are higher than the calculated ones. This implies that the diffusion of Ge into the film took place more intensively at higher growth temperatures.
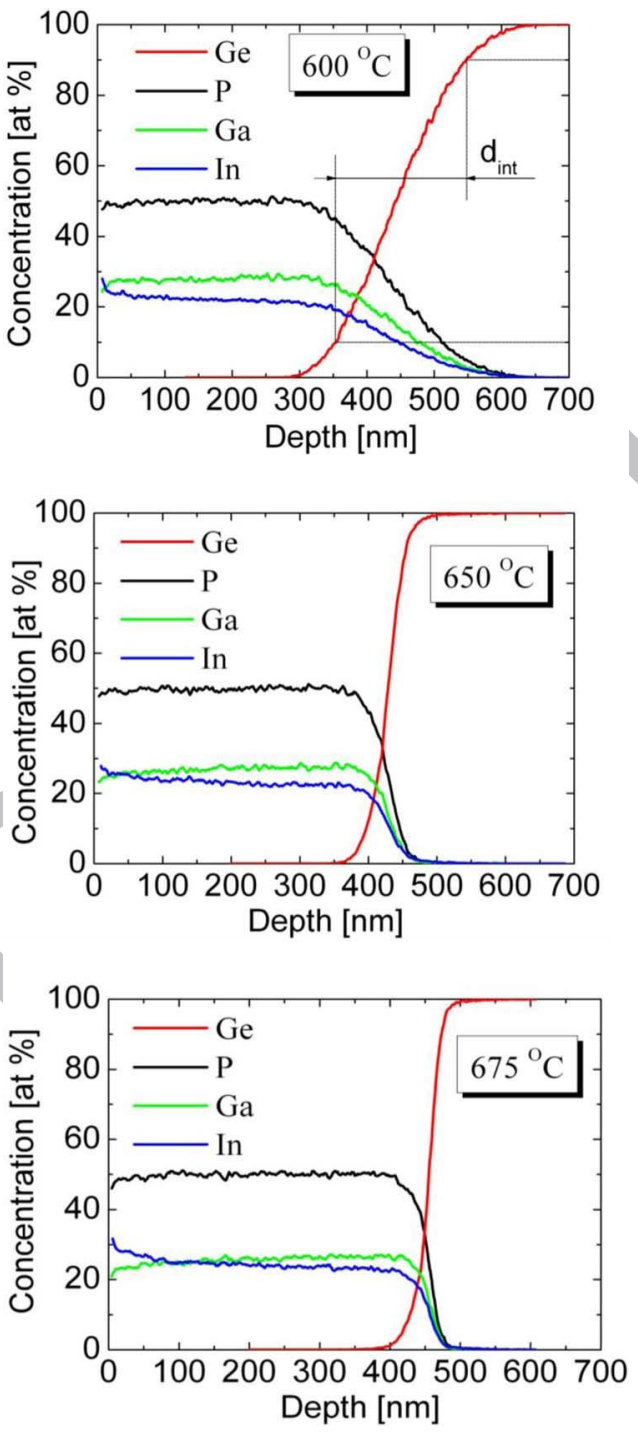

Figure 3. SNMS profiles for the InGaP/Ge sample grown at 600,650 and $675^{\circ} \mathrm{C}$. The method of interface thickness $\left(\mathrm{d}_{\text {int }}\right)$ determination for Ge of Sample 1 is shown in the upper figure.

The volume diffusion and grain boundary diffusion compete with each other in the diffusion mechanism accompanied by MOVPE growth process. The linear fittings of the tails of $\ln C$ vs. $d^{2}$ and $\ln \mathrm{C}$ vs. $\mathrm{d}^{6 / 5}$ functions, where $\mathrm{C}$ is the concentration and $\mathrm{d}$ is the depth as given in Fig. 4 which yield a possibility to estimate the diffusion coefficients. ${ }^{1,15}$ While $\ln \mathrm{C}$ vs. $\mathrm{d}^{2}$ function gives information about the effective diffusivity, the linear part of $\ln \mathrm{C}$ vs. $\mathrm{d}^{6 / 5}$ curve determines the grain boundary diffusion coefficient. The effective diffusion coefficient of Ge in InGaP layer was estimated to be $3.7 \cdot 10^{-23} \mathrm{~m}^{2} / \mathrm{s}$ in case of Sample 2, and $1,5 \cdot 10^{-22} \mathrm{~m}^{2} / \mathrm{s}$ in case of Sample 3. We also found that the linear part of $\ln \mathrm{C}$ vs. $\mathrm{d}^{6 / 5}$ function was longer seen in Sample 3 than in Sample 2, i.e. the effect of grain boundary diffusion was higher in Sample 3 than in Sample 2. The results of Sample 1 could not be used to determine the diffusion coefficient because the thickness of the interface layer was so high that the information concerning to the diffusion was totally smeared out. A deeper understanding of the diffusion process took place during preparation requires more experiments. Our results showed that a diffusion controlled doping effect can exist only when the substrate is kept at high temperature during preparation.

Diffusion of film constituents into the opposite direction, i.e. into the substrate, also exists with lower intensity because the substrate is a 214 215 216 217 218 218 219 220 221 222 223 224 225 225 226
227 227 228 229 229 230 231 232 

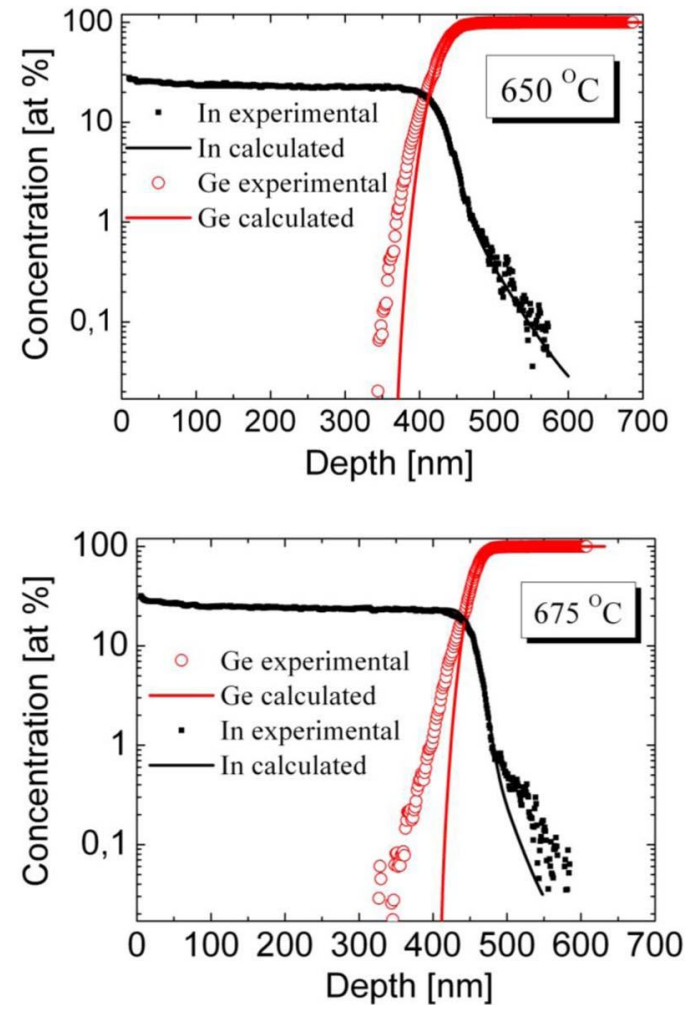

Figure 4. Indium and germanium depth distributions in Samples 2 and 3. The symbols denote the values measured experimentally, the lines are the calculated depth distribution. It can be seen that the diffusion process is more effective at temperature of $675^{\circ} \mathrm{C}$ than it is at $650^{\circ} \mathrm{C}$.

233 single crystal. The indium signal in Fig. 4 and the RBS spectra in Fig. 2 234 represent the result of this process. Although the interface width of the 235 sample grown at $650^{\circ} \mathrm{C}$ was higher than that of the Sample 3 grown 236 at $675^{\circ} \mathrm{C}$, the diffusion was more intensive at higher temperature. 237 This result supports our conclusion that the interface width is mainly 238 determined by the surface roughness and not by the diffusion.

The largest interface width of $195 \mathrm{~nm}$ was found in sample 1 . The explanation for that lies in the crystal structure. The InGaP layer grown at $600^{\circ} \mathrm{C}$ has an ordered phase. ${ }^{9}$ Fig. 5a shows the TEM image of sample grown at $600^{\circ} \mathrm{C}$ taken in cross section, i.e. by viewing the sample along $\quad 011 \square$ direction. It was taken in $220 \square$ bright field mode
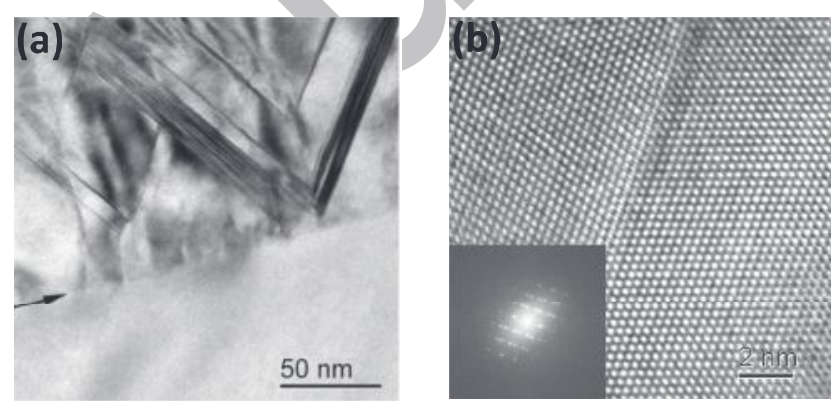

Figure 5. a) TEM image of the sample grown at $600^{\circ} \mathrm{C}$ taken in cross section; b) HRTEM image of a twin in the top part of the layer. Inset: Diffraction pattern extracted by fast Fourier transform of the image, showing the twin $1 / 3$ extra spots along the $\square 11 \square$ direction perpendicular to the twin plane. of operation of TEM. The interface was indicated by the black arrow. ${ }^{244}$ The defects shown in the image were stacking faults and twins lying on 245 (111) planes inclined $54.7^{\circ}$ to the (100) plane. The HR-TEM image of 246 a twin is shown in Fig. 5b. Such defects in the InGaP film originated at 247 the Ge surface, but sometimes quite far $(30-50 \mathrm{~nm})$. During sputtering 248 the surface of this region is rough, the interface widens due to the twin 249 structure, and the surface roughness is non-Gaussian type preventing 250 the calculation of depth distribution.

\section{Conclusions}

InGaP layers grown by MOVPE were characterized to understand 253 the diffusion mechanism between the film and substrate. Surface anal- 254 ysis by AFM showed a temperature dependency of surface roughness. 255 The roughness decreased with the increase of growth temperature. 256 SNMS technique reported the depth distribution of elements and re- ${ }_{257}$ vealed that the interface width decreased with decreasing the surface 25 roughness. Supplementary measurements with calculations based on 25 surface roughness showed that Ge diffused in the film more intensively 260 at higher temperature. Diffusion of film constituents into the opposite 2 direction, i.e. into the substrate, can also exist. We demonstrated that although the interface width of the sample grown at $650^{\circ} \mathrm{C}$ was higher than that of the sample grown at $675^{\circ} \mathrm{C}$, the diffusion was more intensive at the higher temperature, i.e. the interface width is determined by the surface roughness and not by the growth temperature.

\section{Acknowledgments}

One of the authors Joice Sophia Ponraj worked with the support of "ICTP Programme for Training and Research in Italian Laboratories, Trieste, Italy" and gratefully acknowledges the funding agency ICTP and IMEM-CNR, Parma. AFM measurements were acquired at "Centro Interfacoltà Misure" of Parma University. Some part of this work (SNMS measurements) was supported by the TAMOP 4.2.2.A$11 / 1 / K O N V-2012-0036$ project, which is co-financed by the European Union and European Social Fund, as well as by OTKA under grant \#NF 101329. We thank Z. Erdélyi for very useful discussions. The work has been supported by the Scientific Cooperation Agreement between CNR (Italy) and MTA (Hungary).

\section{References}

1. M. Razeghi, The MOCVD Challenge: A survey of GaInAsP-GaAs for photonic and electronic device applications, Vol. 2, pp. 22-49, Institute of Physics Publishing, Bristol/Philadelphia (1995).

2. Y. C. Lee, Y. Zhang, H. J. Kim, S. Choi, Z. Lochner, R. D. Dupuis, J.-H. Ryou, and S.-C. Shen, Electron Devices, IEEE Transactions on, 57, 2964 (2010).

3. Y. C. Hsieh, E. Y. Chang, S. S. Yeh, C. W. Chang, G. L. Luo, C. Y. Chang, and L. C. Ting, J. Cryst. Growth, 289, 96 (2006).

4. T. Nakano, T. Shioda, E. Abe, M. Sugiyama, N. Enomoto, Y. Nakano, and Y. Shimogaki, Appl. Phys. Lett., 92, 112106 (2008).

5. P. E. Smith, M. Lueck, S. A. Ringel, and L. J. Brillson, J. Vac. Sci. Technol., B26, 89 (2008).

6. M. Bosi and C. Pelosi, Progress in Photovoltaics: Research and Applications, 15, 51 (2007).

7. R. R. King, D. Bhusari, D. Larrabee, X.-Q. Liu, E. Rehder, K. Edmondson, H. Cotal, R. K. Jones, J. H. Ermer, C. M. Fetzer, D. C. Law, and N. H. Karam, Progress in Photovoltaics: Research and Applications, 20, 801 (2012).

8. G. Attolini, M. Bosi, M. Calicchio, C. Ferrari, C. Frigeri, K. Vad, A. Csik, and Z. Zolnai, Proceedings of the EW-MOVPE XIII, p. 301, Ulm, Germany (2009).

9. G. Attolini, C. Bocchi, F. Germini, C. Pelosi, A. Parisini, L. Tarricone, R. Kudela, and S. Hasenohrl, Materials Chemistry and Physics, 66, 246 (2000).

10. F. Pászti, A. Manuaba, C. Hajdu, A. A. Melo, and M. F. Da Silva, Nuclear Instruments and Methods in Physics Research Section B: Beam Interactions with Materials and Atoms, 47, 187 (1990).

11. M. Bosi, G. Attolini, C. Ferrari, C. Frigeri, M. Calicchio, F. Rossi, K. Vad, A. Csik, and Z. Zolnai , J. Cryst. Growth, 318, 367 (2011).

12. S. Hofmann, Surf. Interface Anal., 27, 825 (1999).

13. S. Hofmann, Rep. Prog. Phys., 61, 827 (1998).

14. T.-Y. Seong, J. H. Kim, Y.-S. Chun, and G. B. Stringfellow, Appl. Phys. Lett., 70, 3137 (1997) 